\title{
Uma Proposta de Classificação para Internet das Coisas na Educação
}

\author{
Jefferson de Carvalho Cabral, Obionor O. Nóbrega, Fernando A. Aires Lins
}

Departamento de Estatística e Informática - Universidade Federal Rural de Pernambuco (UFRPE) - Rua Dom Manoel de Medeiros, s/n, Dois Irmãos - CEP: 52171-900 Recife - PE

jcabral1500@gmail.com, \{obionor.nobrega, fernandoaires\}@ufrpe.br

\begin{abstract}
The networks intelligent connectivity and ubiquitous computing is a key part of the Internet of Things (IoT). Therefore, in order for the Internet of Things to be successful, the computing paradigm will have to go beyond traditional mobile computing scenarios, and evolve to connect existing objects every day, as well as incorporate intelligence into our environment, comparing the way we learn today. These new forms of learning bring with them great challenges due to IoT's dimension, complexity and various purposes in the educational area. This work proposes an Internet classification of Things in Education, making it possible to identify its impact, its actors, services, objects and the interaction between them.
\end{abstract}

Resumo. A conectividade inteligente entre as redes existentes e a computação ubíqua é uma parte fundamental da Internet das Coisas (IoT). Portanto, para ser bem-sucedido, o paradigma da computação precisará ir além dos cenários tradicionais de computação móvel, e evoluir para conectar os objetos existentes do dia a dia, assim como incorporar inteligência em nosso ambiente, modificando a forma como aprendemos hoje. Estas novas formas de aprendizado trazem grandes desafios devido à complexidade e os mais diversos propósitos de atuação da IoT na área educacional. Este trabalho propõe uma classificação para IoT na Educação, possibilitando identificar qual o seu impacto, seus atores, serviços, objetos e a interação entre eles.

\section{Introdução}

A habilidade de conectar pessoas em qualquer parte do mundo fez da Internet o elemento chave em uma das maiores revoluções tecnológicas do mundo moderno. Esta habilidade está em constante mudança, criando novos conceitos e quebrando velhos paradigmas, possibilitando estar conectado em todos os lugares, a todo o momento e utilizando diversas tecnologias [Gubbi et al. 2013][Matthew e Nitha 2016].

O expressivo desenvolvimento das tecnologias da informação, atrelado ao surgimento e a popularização dos dispositivos móveis e suas aplicações têm transformado a forma com que os usuários recebem, utilizam e salvam as informações. Além disso, a expansão das redes sem fio fornece novas oportunidades e infraestruturas para que indivíduos e instituições sejam capazes de utilizar novos recursos de TI eficientemente [Saritas 2015]. Isso permitirá que cada vez mais objetos presentes em 
nosso cotidiano estejam conectados conosco por meio da Internet. Esta nova evolução da Internet, chamada de Internet do Futuro [Tronco 2010], traz consigo novos desafios como sistemas e infraestrutura centrada no usuário, uma internet centrada em conteúdo; e todas as coisas interconectadas, também chamada de Internet das Coisas (IoT Internet of Things) [Mendonça 2016].

Hoje, o paradigma emergente da Internet das Coisas está sendo visto como protagonista da atual revolução tecnológica em que vive a era da computação. Neste paradigma, os objetos do cotidiano estão conectados, visando à criação de ambientes inteligentes [Gubbi et al. 2013]. Assim como a Internet fez, a IoT tem a capacidade de mudar o modo e os métodos com que as pessoas trabalham, vivem e aprendem novos conceitos [Zhang 2012]. Ao fim do ano de 2017, estima-se que 25 bilhões de "objetos inteligentes" conectados serão implantados e isso irá expandir para 50 bilhões em 2020, demostrando o rápido crescimento e adoção deste novo paradigma, segundo previsão das empresas Cisco e Ericsson [Pruet et al. 2015; Salis et al. 2015].

Com a quantidade de dispositivos conectados e serviços Web robustos na IoT, o mundo hoje tem um novo grande recurso para mudar o processo educacional beneficiando as gerações atuais e futuras [Cornel 2015]. De acordo com [Bhatt e Bhatt 2017], a Internet das Coisas é uma tecnologia emergente e exercerá um papel fundamental em diversos campos como: saúde, transporte, agricultura, segurança e educação.

Estas novas formas de aprendizado, trazem consigo grandes desafios devido à dimensão, a complexidade e os mais diversos propósitos a qual a IoT destina-se a atuar na área educacional. Para se identificar qual o impacto da Internet das Coisas na educação, seus atores, serviços, objetos e a interação entre eles, torna-se essencial a necessidade de uma classificação [Ning e $\mathrm{Hu}$ 2012]. Este artigo tem por objetivo apresentar uma proposta de classificação para Internet das Coisas na Educação, com base nos objetivos que os trabalhos na literatura alcançaram até o momento.

O restante do artigo está organizado da seguinte forma: A Seção 2 apresenta as principais classificações da IoT na literatura; Na Seção 3 são apresentados os principais trabalhos relacionados a Internet das Coisas na Educação; Na Seção 4 é proposto um modelo de classificação de IoT na Educação; e, por fim, na Seção 5 é exposto a conclusão deste trabalho.

\section{A Internet das Coisas e suas Classificações}

Para uma melhor compreensão dos tipos de classificações já existentes na área de Internet das Coisas, nesta seção serão apresentados alguns estudos referentes a estas classificações. Diante do vasto cenário existente para as aplicações da Internet das Coisas, diferentes perspectivas foram consideradas.

Segundo Corsaro [Corsaro 2014], a Internet das Coisas pode ser classificada em dois tipos. O primeiro tipo é o Consumer Internet of Things (CIoT) que representa a classe de aplicações orientadas ao consumidor. Nesta classe, os dispositivos inteligentes são direcionados ao consumidor como, por exemplo: refrigeradores, Google Glass etc, caracterizando-se por possuir taxa e volume de dados baixo. O outro tipo é a Industry 
Internet of Things (IIoT) que constitui nas aplicações voltadas para a indústria. Nesta categoria, os dispositivos são as máquinas que operam no âmbito industrial, de transporte, energético, médico, etc., caracterizada por possuir uma elevada taxa e volume de dados.

De acordo com Morgan [Morgan 2015 apud Smutny 2016], a IoT pode ser classificada como Embeddable, Wearable, Holdable e Surroundable considerando o ser humano elemento central. No Embeddable, os dispositivos IoT, de fato, estão presentes no usuário, ou seja, fazem parte do corpo do ser humano, por exemplo: tatuagens, lentes de contato, pílulas com câmera e monitores para chips. Com relação ao Wearable, os objetos IoT como pulseiras, joias e relógios estão sob o usuário para coletar dados. Já no Holdable, os itens da Internet das Coisas estão perto do usuário, ou seja, ao alcance deles e o mesmo faz um esforço para carregá-los. Como smartphones, outros dispositivos portáteis e os aplicativos pertencentes a eles. Por fim, no Surrounddable as coisas da IoT encontram-se ao redor do usuário presentes no mesmo ambiente ele. Por exemplo, um carro que desvia o usuário do tráfego.

No estudo realizado por Das [Das 2013] a Internet das Coisas é categorizada como Internet das Pessoas e Internet das Coisas com e sem endereço IP. Na Internet das Pessoas, todos os dispositivos eletrônicos pessoais como smartphones, tablets, smartwatch e computadores estão conectados à Internet. Na Internet das Coisas sem endereço IP é necessário a existência de um ou vários sistemas dedicados a conectar os dispositivos IoT, RFID padronizado e ativo, sistemas de localização em tempo real e redes de sensores em malha. Dentro da Internet das Coisas com IP, os objetos inteligentes sentem a presença de outros objetos e se comunicam entre si por meio da Internet sem a intervenção humana.

Para classificar as futuras tecnologias da Internet das Coisas, Ning e Hu [Ning e $\mathrm{Hu}$ 2012] propuseram um modelo constituído por quatro dimensões (corpo, processamento, inteligência e sociabilidade) com base na Unit IoT e Ubiquous IoT (U2IoT). A primeira dimensão é denominada de corpo da IoT. Esta assim como na engenharia de hardware engloba todas as variedades de sensores, redes e centros de dados. A segunda dimensão chamada processamento da IoT, pode ser visualizada como a engenharia de software. Nesta, diversas funções como, identificação, codificação, resolução, transmissão, armazenamento, busca, segurança estão inclusas. Na dimensão da inteligência, estão presentes, o gerenciamento avançado da rede, o controle inteligente, a tomada de decisão automática, percepção humana etc. Na dimensão da sociabilidade, a gestão pública, as restrições de ação moral e as leis de regulamentação da IoT estão presentes.

Segundo Thoma [Thoma et al. 2012], os serviços da IoT podem ser classificados em duas dimensões, respectivamente: relacionamento com a entidade física e com base no ciclo de vida. Na primeira dimensão os serviços podem ser: de baixo nível, de recursos, de entidade e integrado. Os serviços de baixo nível são responsáveis por tornar os serviços de recursos, de entidade e integrado acessíveis uns aos outros. Os serviços de recursos proveem as observações que o dispositivo é capaz de originar ou fornecer as ações necessárias que um recurso é capaz de executar. Os serviços de entidade são fornecidos pelas entidades como composições dos serviços de baixo nível. Os serviços 
VI Congresso Brasileiro de Informática na Educação (CBIE 2017)

Anais dos Workshops do VI Congresso Brasileiro de Informática na Educação (WCBIE 2017)

integrados trabalham com os serviços de entidade e os compõem com serviços não relacionados. Com base no ciclo de vida, os serviços podem ser: implementável, implantado e operacional. Um serviço implementável é um serviço que ainda não está em campo, mas a descrição do acordo de serviço existe em no repositório. Um serviço implantado, já está em campo, no entanto não está habilitado para uso, pois necessita que etapas técnicas ou econômicas sejam concluídas para torná-lo operacional. Por fim, o serviço operacional já se encontra implantado.

A seguir na Tabela 1 será apresentado um resumo dos principais autores e suas respectivas classificações.

Tabela 1. Classificação da Internet das Coisas

\begin{tabular}{|c|c|c|c|c|}
\hline & Classificação & Centrado & Abrangência & Exemplos \\
\hline \multirow[b]{2}{*}{ [Corsaro 2014] } & Consumidor IOT & Usuário & Pessoal & Google Glass \\
\hline & Industria IOT & Objeto & Ambiente & \begin{tabular}{|c|} 
Semáforos \\
inteligentes, Smart \\
Grid
\end{tabular} \\
\hline \multirow{4}{*}{$\begin{array}{c}\text { [Morgan } 2015 \text { apud } \\
\text { Smutny 2016] }\end{array}$} & Embutido & Usuário & Interno & Tatuagens, Lentes \\
\hline & Vestivel & Usuário & Externo & Pulseiras, Relógios \\
\hline & Segurável & Usuário & Próximo & Smartphone \\
\hline & Ambiente & Usuário & Ambiente & Carro, Sala \\
\hline \multirow{2}{*}{ [Das 2014] } & Coisas & Objeto & Ambiente & Sensores \\
\hline & Pessoas & Usuário & Próximo & Tablets \\
\hline \multirow{4}{*}{ [Ning e Hu 2012] } & Corpo & Objeto & Hardware & Sensores \\
\hline & Processamento & Objeto & $\begin{array}{c}\text { Software de baixo } \\
\text { nivel }\end{array}$ & $\begin{array}{l}\text { Identificação, } \\
\text { Transmissão }\end{array}$ \\
\hline & Inteligência & Objeto & $\begin{array}{c}\text { Software de alto } \\
\text { nivel }\end{array}$ & $\begin{array}{c}\text { Software para } \\
\text { tomada de decisão }\end{array}$ \\
\hline & Sociabilidade & Objeto & - & Leis regulamentação \\
\hline \multirow{7}{*}{ [Thoma et al. 2012] } & Baixo nível & Serviço & Software & - \\
\hline & Recursos & Serviço & Software & - \\
\hline & Entidade & Serviço & Software & - \\
\hline & Integrado & Serviço & Software & - \\
\hline & Implementável & Serviço & Software & - \\
\hline & imlantado & Serviço & Software & - \\
\hline & Operacional & Serviço & Software & - \\
\hline
\end{tabular}

Na Tabela 1, além das classificações propostas, pode-se observar quais são os elementos centrais considerados em cada classificação como: o usuário (ser humano), os objetos (coisas) e os serviços. Ressalta-se também a abrangência espacial que cada classificação trata em suas respectivas análises. Além disso, alguns exemplos são apresentados para melhor compreensão dos objetos envolvidos em cada classificação.

\section{A Internet das Coisas na Educação}

Até o presente momento, o crescimento da Internet das Coisas tem atuado positivamente no setor da educação e, da forma com que a tecnologia está sendo utilizada, a mudança ocorre lentamente, desde aplicações estáticas até salas de aulas interativas [Kusmin 
VI Congresso Brasileiro de Informática na Educação (CBIE 2017)

Anais dos Workshops do VI Congresso Brasileiro de Informática na Educação (WCBIE 2017)

2017]. Nesta seção são apresentados os principais trabalhos relacionados a IoT na área educacional.

Um estudo de caso para demonstração dos benefícios da IoT por meio da criação de um laboratório virtual on-line é proposto por Cornel [Cornel 2015]. O desenvolvimento do laboratório virtual on-line é realizado utilizando a plataforma de desenvolvimento de baixo custo Arduino, juntamente com web service gratuito Xively, visando medir a temperatura de um ambiente. Segundo o autor, a Internet das Coisas tem a capacidade de fornecer ensino à distância mesmo para especializações técnicas a partir da criação desses laboratórios.

A existência de uma dependência entre a Computação em Nuvem, a IoT e o Conectivismo, e que a junção desses três paradigmas pode contribuir para o processo de aprendizagem em rede é apresentado por [Saritas 2015]. O conectivismo é uma teoria de aprendizagem que defende a ideia de que o conhecimento existe no mundo e este só pode ser adquirido quando o indivíduo percorre os nós existentes fora da sua própria rede de conhecimento. De acordo com o autor, a Computação em Nuvem será parte vital da IoT considerando que o grande volume de dados seja suportado e processado por esta infraestrutura. A IoT deverá se responsabilizar em conectar os mais diversos objetos inteligentes independente de sua natureza e o Conectivismo fornecerá suporte a todo o fundamento teórico da aprendizagem em rede. Com base na união dos paradigmas o autor garante que é possível elaborar ambientes inteligentes para estimular e fornecer melhores recursos de aprendizagem amplamente acessíveis e experiências em tempo real.

A criação de uma "caixa inteligente" a partir da IoT, do Living Labs e do iLearning, considerado um dos seis pilares do iCampus, foi proposta por Chin [Chin e Callaghan 2013]. Após o desenvolvimento da caixa, utilizaram o paradigma Pervasive Interactive Programming (PiP) juntamente com a IoT para ensinar habilidades básicas de programação a alguns grupos de alunos e funcionários de uma escola. Os autores percebem a IoT como provedora de recursos físicos (sensores e celulares), e como um framework para ensino.

As técnicas de aprendizagem interativas para alunos que frequentam a escola como: Aprendizagem de forma associativa, Servidores de laboratórios centralizados e Análise constante do progresso do aluno são apresentadas por Rahman [Rahman et al. 2016]. Simultaneamente também são mostradas as técnicas de aprendizagem para estudantes que não possuem condições de comparecer a uma sala de aula, por exemplo: Gamificação de testes e atribuições, Redes sociais como meio educacional, Conectividade Escola-Casa e Sala de aula virtual. As mudanças para as tecnologias educacionais conduzidas pela Internet das Coisas como: a adição de ativos para ensino, as ferramentas de publicação, os canais de comunicação, o aumento na eficácia do treinamento prático, a redução nos espaços de aprendizagem e o aprimoramento do modelo de ensino teórico são apontadas por [Zhang 2012; Rahman et al. 2016].

Um sistema para gerenciamento de livros dentro da biblioteca utilizando a tecnologia de Near Field Communication (NFC) foi proposto por Matthew [Matthew e Nitha 2016]. Além da capacidade de reconhecer e atualizar o banco de dados quando 
um livro é retirado ou devolvido à prateleira, o sistema conta com um alarme sonoro ao tentar devolver o livro em uma posição diferente da origem, provê um resumo detalhado sobre o livro retirado por meio da existência de um alto falante presente na prateleira, oferece recomendações livros com características semelhantes aos livros já retirados pelo usuário, notifica o usuário assim que um novo livro está disponível e por fim, toda a troca de mensagens entre o sistema e os dispositivos é criptografada. Segundo os autores, a IoT é capaz de aumentar a educação e facilitar a vida acadêmica por meio da automatização de ambientes.

Uma visão geral das soluções "inteligentes" (Educação Inteligente, Ensino Inteligente, Aprendizagem Inteligente, Sala de Aula Inteligente e Escola Inteligente) é apresentada por Kusmin [Kusmin 2017]. Segundo o autor, a Internet das Coisas pode fornecer aos alunos atividades envolventes e de alta qualidade a partir do uso de smartphones, tablets, quadros inteligentes e aplicativos.

Um novo conceito chamado Universidade Inteligente é descrito por Catã [Catã 2015]. Com base nas necessidades e vantagens desse conceito, o autor apresenta a criação de uma possível arquitetura para as futuras salas de aula inteligentes composta por objetos inteligentes. $\mathrm{O}$ autor acredita na criação de uma sala de aula inteligente com intuito de reduzir a distração dos estudantes com o celular.

A descrição de um sistema IoT para o aprendizado da Língua Brasileira de Sinais (Libras) é proposto por Silva [Silva, Lima e Bastos 2016]. Esse sistema possui um tutor inteligente capaz de ensinar como o sinal de Libras deve ser reproduzido. Os autores consideram que a aplicação contém três elementos da IoT como: sensoriamento, semântica e dados. O processo de sensoriamento é executado pela weareable computing que é utilizada para captura dos movimentos dos braços e mãos por meio do uso de luvas eletrônicas durante a execução dos gestos dos sinais de Libras. Os gestos retidos serão repassados para a aplicação que será executada em outro dispositivo weareable, um SmartGlass. Os processos de semântica e dados consistem na interpretação correta do gesto com objetivo de comparar o gesto produzido com gesto existente em um banco de dados e assim fornecer um resultado adequado ao usuário.

Com objetivo de identificar as soluções IoT desenvolvidas para o campo da Educação a Distância (EAD), um Mapeamento Sistemático foi realizado por Fuzeto [Fuzeto e Braga 2016]. O resultado do trabalho apresenta os tipos de ambientes de EAD, por exemplo: E-learning, M-learning e U-learning, tipos de recursos educacionais utilizados como: imagem, livros, vídeos, e tecnologias IoT empregadas como: etiquetas RFID, e Redes de Sensores Sem Fio (RSSF).

Com intuito de viabilizar as experiências práticas no ensino de Física e Geologia na Universidade Federal do Espírito Santo (UFES), Oliveira [Oliveira et al. 2016] sugere o projeto e implementação de uma estação geomagnética aplicando componentes de custo reduzido como: o Raspberry $\mathrm{Pi}$, tecnologias de código fonte aberto por exemplo: o Linux Raspbian Jessie e o emprego da IoT que possibilita acesso aos dados gerados por meio de uma interface Web. 
VI Congresso Brasileiro de Informática na Educação (CBIE 2017)

Anais dos Workshops do VI Congresso Brasileiro de Informática na Educação (WCBIE 2017)

Baseado na análise destas referências bibliográficas, foi possível identificar que a IoT não será apenas responsável por fornecer ambientes inteligentes no campo educacional, mas ela permitirá facilitar a vida de professores e alunos, prover maior interação entre eles, gerar conteúdo de ensino personalizado, permitir a automatização de tarefas consideradas impeditivas no cotidiano educacional do professor, viabilizar feedbacks instantâneos para os alunos durante a realização das provas de conhecimento, extensão dos ambientes de aprendizagem, entre outros.

Com intuito de facilitar a visualização sobre o que a Internet das Coisas está proporcionando ao campo educacional, é apresentado a seguir na Tabela 2 o objetivo da IoT na educação visto em cada artigo avaliado.

Tabela 2. Objetivos da loT na Educação

\begin{tabular}{|c|c|c|c|}
\hline & Título & Objetivo & Elementos \\
\hline [Cornel 2015] & $\begin{array}{l}\text { O papel da Internet das Coisas para o } \\
\text { Fornecimento Contínuo da Educação }\end{array}$ & $\begin{array}{l}\text { Criar um laboratório virtual online para } \\
\text { fornecimento de ensino à distância. }\end{array}$ & $\begin{array}{c}\text { Web Service Xively e Arduino, } \\
\text { Laboratório Virtual Online }\end{array}$ \\
\hline [Saritas 2015] & \begin{tabular}{|c|} 
Os Paradigmas Tecnológicos e Teóricos \\
Emergentes na Educação: A interrelação \\
entre Computação em Nuvem, Internet das \\
Coisas e Conectivismo \\
\end{tabular} & $\begin{array}{c}\text { Elaborar ambientes inteligentes para } \\
\text { estimular e fornecer melhores recursos } \\
\text { de aprendizagem. }\end{array}$ & Computação em Nuvem \\
\hline $\begin{array}{l}\text { [Chin e Callaghan } \\
\text { 2013] }\end{array}$ & $\begin{array}{c}\text { Laboratório de Vivência Educacional: Uma } \\
\text { nova abordagem baseada em Internet das } \\
\text { Coisas para Ensino e Pesquisa }\end{array}$ & $\begin{array}{l}\text { Prover recursos físicos e servir de } \\
\text { framework para ensino. }\end{array}$ & $\begin{array}{l}\text { Smart Box, iCampus, Living Labs, } \\
\text { Smartphones, Sensores }\end{array}$ \\
\hline $\begin{array}{l}\text { [Rahman et al. } \\
\text { 2016] }\end{array}$ & $\begin{array}{c}\text { TIC e Internet das Coisas para Criar } \\
\text { Ambiente de Aprendizagem Inteligente } \\
\text { para Estudantes em Institutos de } \\
\text { Educação na Îndia }\end{array}$ & $\begin{array}{c}\text { Fornecer ferramentas de publicação, } \\
\text { canais de comunicação, redução nos } \\
\text { espaços de aprendizagem e o aumento } \\
\text { na eficácia do treinamento prático. }\end{array}$ & $\begin{array}{c}\text { Realidade Aumentada, Animação 3D, } \\
\text { Laboratórios Virtuais, Smart Board, } \\
\text { Tablets, Jogos Interativos, Redes } \\
\text { Sociais, Redes de Sensores Sem Fio, } \\
\text { Smart Classroom }\end{array}$ \\
\hline $\begin{array}{l}\text { [Matthew e Nitha } \\
\text { 2016] }\end{array}$ & $\begin{array}{l}\text { Universidade Inteligente uma abordagem } \\
\text { IoT: Uma pesquisa sobre loT na educação }\end{array}$ & $\begin{array}{c}\text { Aumentar a educação e facilitar a vida } \\
\text { acadêmica por meio da automatização } \\
\text { de ambientes }\end{array}$ & $\begin{array}{c}\text { Etiquetas e Leitores RFID, NFC, } \\
\text { Smartphones, Bibliotecas Inteligentes }\end{array}$ \\
\hline [Kusmin 2017] & $\begin{array}{c}\text { Abordagens da Sociedade da Informação e } \\
\text { Processos de TIC }\end{array}$ & $\begin{array}{c}\text { Fornecer aos alunos atividades } \\
\text { envolventes e de alta qualidade a partir } \\
\text { do uso de smartphones tablets, } \\
\text { quadros inteligentes etc. }\end{array}$ & $\begin{array}{c}\text { Smartphones, Smart Tablets, Smart } \\
\text { Boards, Smart Classroom }\end{array}$ \\
\hline [Catã 2015] & $\begin{array}{c}\text { Universidade Inteligente, um novo conceito } \\
\text { na Internet das Coisas }\end{array}$ & $\begin{array}{c}\text { Desenvolver uma sala de aula } \\
\text { inteligente com intuito de reduzir a } \\
\text { distração dos alunos em sala de aula. }\end{array}$ & $\begin{array}{c}\text { Leitor RFID, Etiquetas NFC, Smart } \\
\text { Classroom, Smartphone, Web Service } \\
\text { Xively, Arduino, Raspberry Pi, PCDuino } \\
\text { BeagleBone }\end{array}$ \\
\hline $\begin{array}{c}\text { [Silva, Lima e } \\
\text { Bastos 2016] }\end{array}$ & $\begin{array}{c}\text { Aperfeiçoando o aprendizado da Libras } \\
\text { utilizando elementos de Internet das } \\
\text { Coisas }\end{array}$ & $\begin{array}{c}\text { Descrever um sistema computacional } \\
\text { para aperfeiçoar o aprendizado de } \\
\text { Libras }\end{array}$ & $\begin{array}{l}\text { Luvas Eletrônicas, WiFi, Bluetooth, } \\
\text { Smartphones, Óculos Virtuais, Android }\end{array}$ \\
\hline $\begin{array}{c}\text { [Fuzeto e Braga } \\
\text { 2016] }\end{array}$ & $\begin{array}{c}\text { Um Mapeamento Sistemático em } \\
\text { Progresso Sobre a Internet das Coisas e } \\
\text { Educação à Distância }\end{array}$ & $\begin{array}{c}\text { Demonstrar as soluções loT } \\
\text { desenvolvidas para EAD e as soluções } \\
\text { que não são explicitamente entendidas } \\
\text { como soluções loT. }\end{array}$ & $\begin{array}{c}\text { U-learning, M-learning, E-learning, } \\
\text { Etiquetas RFID, Redes de Sensores } \\
\text { Sem Fio, Smartphones, PDAs, Cloud } \\
\text { Centers }\end{array}$ \\
\hline $\begin{array}{l}\text { [Oliveira et al. } \\
\text { 2016] }\end{array}$ & $\begin{array}{c}\text { Uma Estação de Medição Geomagnética } \\
\text { de Acesso Ubiquo para Estudos de } \\
\text { Geofísica }\end{array}$ & $\begin{array}{l}\text { Apresentar o projeto e a } \\
\text { implementação de uma estação } \\
\text { geomagnética com o uso de } \\
\text { componentes de baixo custo e } \\
\text { tecnologias abertas. }\end{array}$ & $\begin{array}{c}\text { Sensores Fluxgate, Raspberry Pi } 3 \text {, } \\
\text { Linux Raspian Jessie }\end{array}$ \\
\hline
\end{tabular}

Na Tabela 2 os diversos propósitos da IoT no âmbito educacional identificados tem como objetivo demonstrar a diversidade de soluções existentes e a dimensão da Internet das Coisas neste meio. Além disso, foi apresentado uma variedade de tecnologias e elementos que compõe as soluções de IoT. Acredita-se que a necessidade do surgimento de novas tecnologias e a utilização destas para prover uma maior interação entre objetos tornando-os menos dependentes dos seres humanos induzirá ainda mais o crescimento da IoT de uma forma geral. 
VI Congresso Brasileiro de Informática na Educação (CBIE 2017)

Anais dos Workshops do VI Congresso Brasileiro de Informática na Educação (WCBIE 2017)

\section{Proposta de Classificação para IoT na Educação}

A proposta de classificação da Internet das Coisas na Educação é baseada em quatro dimensões que interagem entre si, podendo ser desenvolvida soluções específicas e individuais para uma dimensão, ou para um conjunto destas dimensões:

-Dimensão Humana: São todos os elementos humanos presentes no contexto educacional capazes de se beneficiar da utilização da IoT como:

o Aluno presencial - é todo aluno que, presencialmente, utiliza o conceito de "coisas" para adquirir conhecimento em qualquer lugar e a qualquer momento.

o Aluno virtual - é todo aluno que, virtualmente, utiliza objetos ou ambientes remotos para adquirir conhecimento.

o Professor - é todo ser humano capaz que interferir no aprendizado, manipulação, desenvolvimento ou publicação de dados e informações para um melhor aprendizado.

-Dimensão Física: São todos os elementos não humanos existentes no âmbito educacional, ou seja, é a infraestrutura desenvolvida a partir da utilização da IoT e suas tecnologias. Esses elementos podem ser do tipo:

○Objeto (ou coisas): - Elementos físicos voltados para o fornecimento informações de/para ensino e aprendizagem. Em grande parte, os elementos físicos trocam/coletam dados a partir de bancos de dados massivos distribuídos ou de ambientes externos, e são compostos por:

- Software: sistemas operacionais, aplicativos específicos para IoT.

-Hardware: micro controladores e interface de conectividade de rede utilizadas em IoT.

○ Ambiente: é considerado um ambiente de IoT na Educação um conjunto de objetos inteligentes em uma restrita área geográfica, que interagem entre si, a fim de produzir conteúdo relevante, seja para outro elemento físico ou para um elemento humano. As salas de aula inteligentes, laboratórios e as bibliotecas inteligentes são bons exemplos de Ambientes IoT na educação.

o Ecossistemas: é formado um conjunto de ambientes interligados entre si, que proporcionam a troca de informações com o objetivo de um melhor aprendizado de um elemento humano. Destacam-se como exemplo as Universidades Inteligentes, Campus Inteligente e Prédios Inteligentes.

-Dimensão de Conteúdo: São todas as informações relevantes geradas após a troca de dados entre objetos, entre um objeto e conjunto de objetos e/ou um objeto com uma fonte de dados externa como: banco de dados, internet, serviços de nuvem e outros ambientes externos. Esta dimensão tem como função a coleta, o armazenamento, a disponibilização e a troca de dados e informação.

Dimensão de Interação: As trocas de mensagens entre elementos de uma dimensão, ou entre elementos de diferentes dimensões só é possível devido às mensagens ou ações da 
VI Congresso Brasileiro de Informática na Educação (CBIE 2017)

Anais dos Workshops do VI Congresso Brasileiro de Informática na Educação (WCBIE 2017)

dimensão de interação. É nesta dimensão que são desenvolvidas a forma como os conteúdos serão acessados ou trocados, seja por mensagens de requisição e resposta de conteúdo em um banco de dados na nuvem, ou por arquiteturas orientadas a serviço para IoT.

Para uma melhor compreensão dos conceitos descritos na proposta de classificação, a Figura 1 demonstra a interação entre essas quatro classes existentes, onde se verifica a granularidade de cada dimensão.

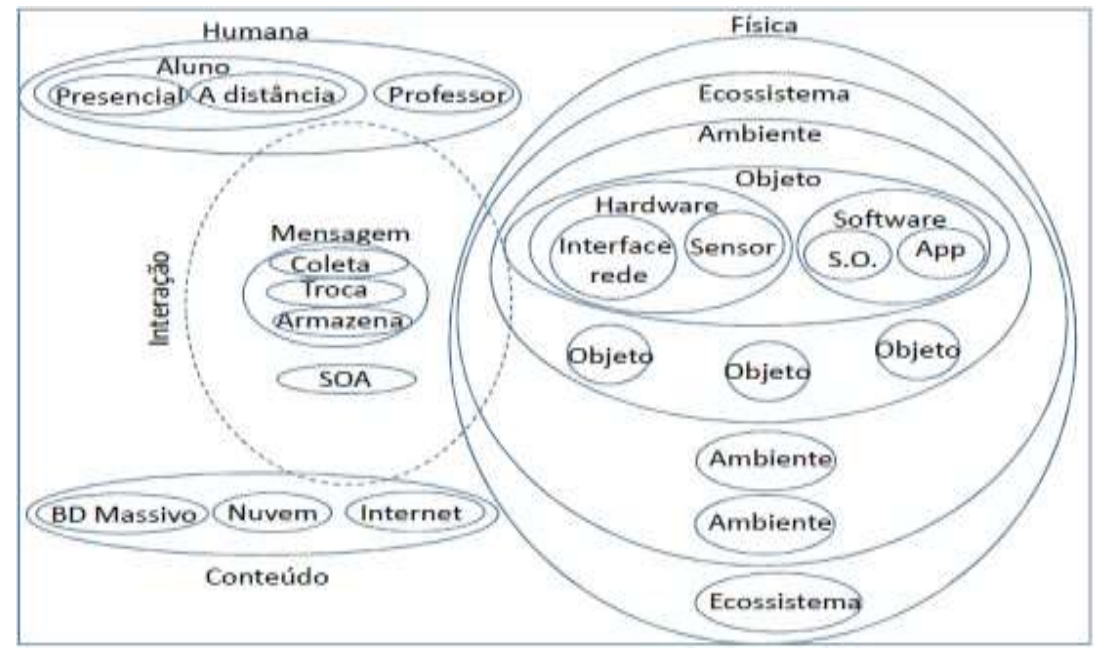

Figura 1. Classificação de Internet das Coisas na Educação.

Uma vez apresentado a proposta de classificação da IoT na educação, é pertinente a elaboração de uma análise dos trabalhos na literatura que tratam de IoT e educação relacionando-os com a classificação proposta. A Tabela 3 apresenta esta análise classificatória, onde se verifica quais dimensões tratam estes trabalhos.

Tabela 3. Classificação dos Trabalhos da loT na Educação

\begin{tabular}{|c|c|c|c|c|c|c|c|}
\hline & \multicolumn{7}{|c|}{ Dimensão } \\
\hline & \multicolumn{2}{|c|}{ Humana } & \multicolumn{3}{|c|}{ Física } & \multirow[t]{2}{*}{ Conteúdo } & \multirow[t]{2}{*}{ Interação } \\
\hline & Aluno & Professor & Objeto & Ambiente & Ecossistema & & \\
\hline [Kusmin 2017] & $X$ & $X$ & $X$ & $X$ & $\mathrm{X}$ & $X$ & \\
\hline [Cornel 2015] & $\mathrm{X}$ & & $\mathrm{X}$ & $\mathrm{X}$ & & & \\
\hline [Saritas 2015] & & & & $\mathrm{X}$ & & $\mathrm{X}$ & $X$ \\
\hline [Chin e Callaghan 2013] & & & $X$ & $\mathrm{X}$ & $x$ & & \\
\hline [Rahman et al. 2016] & $\mathrm{X}$ & & & $X$ & & & \\
\hline [Zhang 2012] & & & $X$ & & & $X$ & $\mathrm{X}$ \\
\hline [Matthew e Nitha 2016] & & & $X$ & $\mathrm{X}$ & & & \\
\hline [Catã 2015] & & & & $\mathrm{X}$ & $x$ & & \\
\hline [Silva, Lima e Bastos 2016] & & & $X$ & & & $X$ & $X$ \\
\hline [Fuzeto e Braga 2016] & $x$ & & $X$ & $\mathrm{X}$ & & & \\
\hline [Oliveira et al. 2016] & & & $x$ & & & $\mathrm{X}$ & $\mathrm{X}$ \\
\hline
\end{tabular}

Como resultado da classificação, verifica-se que nenhum dos trabalhos analisados tangenciam as quatro dimensões propostas, no máximo três delas. Isso pode 
dever-se ao fato de que a IoT ainda é um tema muito recente dentro do campo educacional. Também foi visto que os trabalhos apresentados por [Matthew e Nitha 2016] e [Catã 2015] abrangem apenas a dimensão física, pois um ambiente é composto basicamente de objetos que também pertence a mesma dimensão e um ecossistema que é constituído de ambientes. Na dimensão humana nota-se que a maioria dos trabalhos possui foco no aluno, isso mostra que os autores têm concentrado seus esforços para desenvolver aplicações IoT que visam melhorar as formas atuais de ensino e aprendizagem para esses alunos. Na dimensão física nota-se o equilíbrio existente entre os trabalhos que referenciam os objetos e os ambientes, além da elevada dependência entre esses dois elementos. Isso retrata que a maioria dos autores tem utilizado os objetos para desenvolver ambientes inteligentes com a finalidade de levar o conteúdo além das salas de aulas e laboratórios tradicionais. Os trabalhos relacionados as dimensões de conteúdo e interação mostram que em grande parte é necessário haver interação entre os elementos das outras dimensões para geração de conteúdo. Isso não significa dizer que a dimensão de conteúdo dependa exclusivamente da dimensão de interação e vice-versa, cada dimensão existe separadamente, no entanto devem se relacionar o máximo possível para fornecer uma infraestrutura IoT adequada ao âmbito educacional.

\section{Conclusão}

O tamanho e a complexidade do mundo gerado ao redor da IoT permitiram o surgimento das mais diversas denominações, aplicações e áreas de atuação. Especificamente na educação verificou-se que a IoT beneficiará os principais atores já conhecidos no processo educacional, assim como, será capaz de fornecer ambientes físicos inteligentes de ensino e aprendizagem a partir do emprego e interação entre objetos inteligente e a possibilidade de desenvolvimento de ambientes virtuais com a finalidade de tornar ubíquo o ensino e a aprendizagem, assim como um ecossistema inteligente que forneça novos conhecimentos a um aluno, em qualquer local ou momento.

Com o intuito de delimitar o escopo e o entendimento do significado da Internet das Coisas na educação, apresentou-se uma classificação de IoT na educação baseada nos trabalhos relacionados, onde quatro dimensões foram apresentadas. Espera-se que a classificação proposta neste artigo possa nortear os futuros pesquisadores da área a definir de forma mais clara as aplicações e seus respectivos objetivos no ambiente educacional da Internet das Coisas.

\section{Referências}

BHATT, Juhi; BHATT, Anurag. IoT Techniques to Nurture Education Industry: Scope \& Opportunities. International Journal On Emerging Technologies. Uttarakhand, India, p. 128-132. mar. 2017.

CATÃ, Marian. Smart university, a new concept in the Internet of Things. 2015 14th Roedunet International Conference - Networking In Education And Research (roedunet Ner), Craiova, Romania, p.195-197, set. 2015. IEEE. 
VI Congresso Brasileiro de Informática na Educação (CBIE 2017)

Anais dos Workshops do VI Congresso Brasileiro de Informática na Educação (WCBIE 2017)

CHIN, Jeannette; CALLAGHAN, Vic. Educational Living Labs: A Novel Internet-ofThings Based Approach to Teaching and Research. 2013 9th International Conference On Intelligent Environments, Athens, Greece, p.92-99, jul. 2013. IEEE.

CORNEL, Constantin-eugen. The Role of Internet of Things for a Continuous Improvement in Education. Hyperion Economic Journal. Bucharest, Romania, p. 2431. jun. 2015.

CORSARO, Angelo. What is right messaging data sharing standart for the IoT. 2014. Disponível em: <http://www.prismtech.com/vortex/resources/youtube-videosslideshare/messaging-and-data-sharing-standards-internet-things $>$. Acesso em: 02 jul. 2017.

DAS, Raghu. The Internet of Things: Challenges and Opportunities beyond the hype. 2014. Disponível em: <https://pt.slideshare.net/IT2Industry/id-techexelectronica>. Acesso em: 02 jul. 2017.

FUZETO, Ricardo; BRAGA, Rosana. Um Mapeamento Sistemático em Progresso Sobre Internet das Coisas e Educação à Distância. Anais dos Workshops do V Congresso Brasileiro de Informática na Educação (CBIE 2016), [s.1.], p.1334-1343, 10 nov. 2016. Sociedade Brasileira de Computação - SBC.

GUBBI, Jayavardhana et al. Internet of Things (IoT): A vision, architectural elements, and future directions. Future Generation Computer Systems, [s.1.], v. 29, n. 7, p.1645-1660, set. 2013. Elsevier BV.

HAN, Son N. et al. Service composition for IP smart object using realtime Web protocols: Concept and research challenges. Computer Standards \& Interfaces, [s.1.], v. 43, p.79-90, jan. 2016. Elsevier BV. http://www. researchtrend .net/ ijet/pdf/33-S824.pdf. Acesso em: 02 jul. 2017.

KUSMIN, Marge. Information Society Approaches and ICT Processes. 2017. Disponível em: <http://www.tlu.ee/ pnormak/ISA/Analytical articles/9-Application of the Internet of Things in Education-M.Kusmin.pdf $>$. Acesso em: 12 jun. 2017.

LI, Shancang; XU, Li da; ZHAO, Shanshan. The internet of things: a survey. Information Systems Frontiers, New York, Usa, v. 17, n. 2, p.243-259, 26 abr. 2014. Springer Nature.

MATHEW, Ankitha; P, Nitha K.. Smart Academy an IoT approach: A survey on IoT in education. International Journal Of Advanced Research Trends In Engineering And Technology. Thrissur, India, p. 37-41. fev. 2016.

MENDONÇA, F. F. ; NÓBREGA, Obionor ; CUNHA, P. R. F. . Avaliação do Impacto da Segurança sobre a Fragmentação em Redes de Sensores Sem Fio na Internet das Coisas. In: Simpósio Brasileiro de Redes de Computadores e Sistemas Distribuídos, 2016, Salvador.

NING, Huansheng; HU, Sha. Technology classification, industry, and education for Future Internet of Things. International Journal Of Communication Systems, [s.1.], v. 25, n. 9, p.1230-1241, 17 maio 2012. Wiley-Blackwell. http://dx.doi.org/10.1002/dac.2373. 
VI Congresso Brasileiro de Informática na Educação (CBIE 2017)

Anais dos Workshops do VI Congresso Brasileiro de Informática na Educação (WCBIE 2017)

OLIVEIRA, Fábio et al. Uma Estação de Medição Geomagnética de Acesso Ubíquo para Estudos de Geofísica. Anais dos Workshops do V Congresso Brasileiro de Informática na Educação (CBIE 2016), [s.1.], p.1354-1363, 10 nov. 2016. Sociedade Brasileira de Computação - SBC.

PRUET, Putjorn et al. Exploring the Internet of "Educational Things"(IoET) in rural underprivileged areas. 2015 12th International Conference On Electrical Engineering/electronics, Computer, Telecommunications And Information Technology (ecti-con), Hua Hin, Thailand, p.1-5, jun. 2015. IEEE.

RAHMAN, Munib Ur et al. ICT and internet of things for creating smart learning environment for students at education institutes in India. 2016 6th International Conference - Cloud System And Big Data Engineering (confluence), Noida, India, p.701-704, jan. 2016. IEEE.

SALIS, Carole et al. IoT-DESIR: A case study on a cooperative learning experiment in Sardinia. 2015 International Conference On Interactive Collaborative Learning (ICL), Florence, Italy, p.785-792, set. 2015. IEEE.

SARITAS, Mustafa Tuncay. The Emergent Technological and Theoretical Paradigms in Education: The Interrelations of Cloud Computing (CC), Conectivism and Internet of Things (IoT). Acta Polytechnica Hungarica. Balikesir, Turkey, p. 161-179. jun. 2015. http://www.uni-obuda.hu/journal/Saritas_62.pdf. Acessado em 02/07/2017.

SILVA, Rafael de Amorim; LIMA, Lucas; BASTOS, Rodrigo. Aperfeiçoando o aprendizado de Libras utilizando elementos de Internet das Coisas. Anais dos Workshops do $\mathrm{V}$ Congresso Brasileiro de Informática na Educação (CBIE 2016), [s.1.], p.1364-1373, 10 nov. 2016. Sociedade Brasileira de Computação - SBC.

SMUTNY, Pavel. Different perspectives on classification of the Internet of Things. 2016 17th International Carpathian Control Conference (iccc), Tatranska Lomnica, Slovakia, p.692-696, maio 2016. IEEE.

TEMKAR, Rohini et al. Internet of Things for Smart Classrooms. International Research Journal Of Engineering And Technology (irjet). Chembur, Mumbai, p. 203207. jul. 2016. https://www.irjet.net/archives/V3/i7/IRJET-V3I739.pdf. Acesso em: 02 jul. 2017.

THOMA, Matthias et al. On IoT-services: Survey, Classification and Enterprise Integration. 2012 Ieee International Conference On Green Computing And Communications, Besancon, France, p.257-260, nov. 2012. IEEE. http://dx.doi.org/10.1109/greencom.2012.47.

TROCO, Tania.(2010), New Network Architectures: The Path to the Future Internet. Springer, $1^{\text {th }}$ edition.

ZHANG, Tianbo. The Internet of Things Promoting Higher Education Revolution. 2012 Fourth International Conference On Multimedia Information Networking And Security, Nanjing, China, p.790-793, nov. 2012. IEEE. 